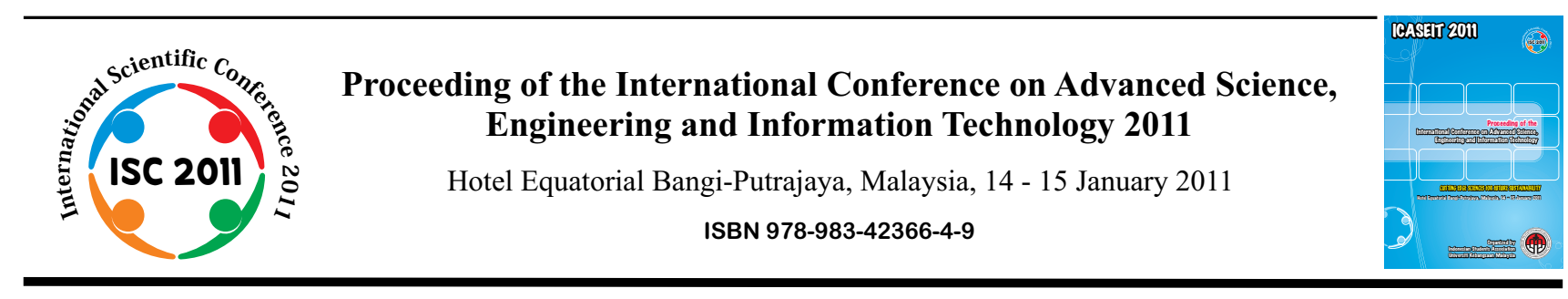

\title{
Intracranial Hemorrhage Annotation for CT Brain Images
}

\author{
Tong Hau Lee ${ }^{\#}$, Mohammad Faizal Ahmad Fauzi ${ }^{*}$, Su-Cheng Haw ${ }^{\#}$

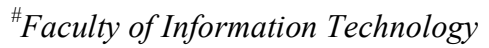 \\ Multimedia University, Jalan Multimedia, 63100 Cyberjaya, Selangor, Malaysia. \\ Tel.:+603-83125404,E-mail: hltong@mmu.edu.my \\ * Faculty of Engineering, \\ Multimedia University, Jalan Multimedia, 63100 Cyberjaya, Selangor, Malaysia. \\ Tel.:+603-8312 5330,E-mail:faizal1@mmu.edu.my
}

\begin{abstract}
In this paper, we created a decision-making model to detect intracranial hemorrhage and adopted ExpectationMaximization(EM) segmentation to segment the Computed Tomography (CT) images. In this work, basically intracranial hemorrhage is classified into two main types which are intra-axial hemorrhage and extra-axial hemorrhage. In order to ease classification, contrast enhancement is adopted to finetune the contrast of the hemorrhage. After that, k-means is applied to group the potential and suspicious hemorrhagic regions into one cluster. The decision-making process is to identify whether the suspicious regions are hemorrhagic regions or non-regions of interest. After the hemorrhagic detection, the images are segmented into brain matter and cerebrospinal fluid (CSF) by using expectation-maximization (EM) segmentation. The acquired experimental results are evaluated in terms of recall and precision. The encouraging results have been attained whereby the proposed system has yielded 0.9333 and 0.8880 precision for extra-axial and intra-axial hemorrhagic detection respectively, whereas recall rate obtained is 0.9245 and 0.8043 for extra-axial and intra-axial hemorrhagic detection respectively.
\end{abstract}

Keywords - Intracranial hemorrhage, CT brain images, image annotation.

\section{INTRODUCTION}

Unequivocally Computed Tomography (CT) is a tool for precise analysis of intracranial hemorrhage. The intracranial hemorrhage is bleeding inside the skull and it can result from head trauma. Basically, intracranial hemorrhage can be divided into two subgroups which are intra-axial and extraaxial. Extra-axial hemorrhage is a type of bleeding that happens outside of the brain tissue. On the other hand, Intraaxial hemorrhage is a type of bleeding that happens inside the brain tissue.

The annotation and auto-detection of hemorrhage never aim to replace the physician by predicting the disease but to assist him/her in diagnosis. To annotate the abnormalities, rule-based approach has been proposed to label the abnormal regions such as calcification, hemorrhage and stroke lesion[1,2]. Y.Liu et al. has developed semantics-based biomedical image indexing and retrieval system[3]. In this system, they proposed a symmetric detection approach to detect the abnormalities. It checks the symmetry for each slice to determine whether the mid line of is shifted or not. If mid line is shifted, the hemorrhage is considered exist. Besides, the similar mid line approach for hemorrhagic detection is also employed by T. Hara et al. [4] and T. Chan[5].

For CT brain images, problems emerged in segmentation are noise, different objects within the same range of intensity, and partial volume effect. Consequently, various segmentation techniques for CT brain images have been developed such as case-based reasoning[6], morphological processing with thresholding[7] and clustering algorithm[8,9] and active contour[10].

This research is an extension of previous efforts[11]. The extended goal for current research is to develop a decisionmaking model for the classification of the extra-axial and intra-axial hemorrhages. 


\section{SYSTEM OVERVIEW}

Generally, the CT brain images will be preprocessed to ease the annotation process as shown in Fig. 1.

The original image is in DICOM format which lacks dynamic range. Therefore, the contrast enhancement is required to increase the visibility of the intracranial area. The enhanced image is used to extract the intracranial area.

Once intracranial area is located, second stage contrast enhancement is applied to enhance the contrast of region of interest(ROI). This is to facilitate the clustering process by k-means method. After clustering, we extract the features from each potential ROI region. Then decision-making process is used to detect the hemorrhage. Lastly, EM segmentation is applied to acquire the CSF and brain matter. The segmented images can be used for the subsequent retrieval and classification works.

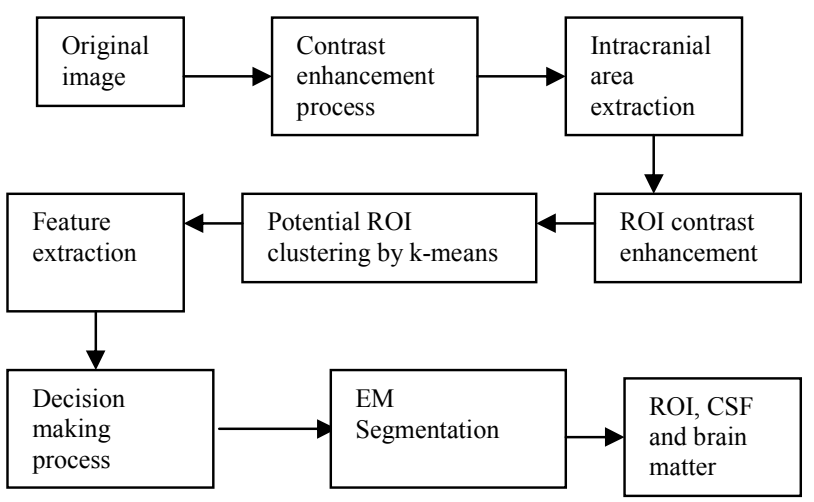

Fig. 1 Flow of the system

\section{CONTRAST ENHANCEMENT PROCESS}

The contrast enhancement is to increase the visibility of the original image. As shown in Fig. 2 only certain parts of the original image appear to be visible.

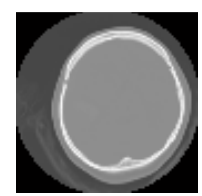

Fig. 2 Example of an original image

Therefore, an auto contrast enhancement system was proposed to improve the visibility of the images. In order to achieve this, the original image will undergo the enhancement process as shown in Fig 3.

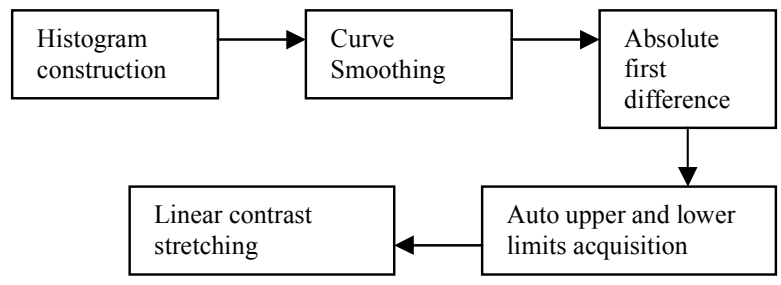

Fig. 3 Contrast enhancement flow
Firstly histogram is constructed as shown in Fig. 4. From Fig. 4, it is consisted of several higher and lower peaks. However, only the rightmost peak is within the significant range for region of interest. Then, for the smoothing process, convolution operation with a vector whose each element is the value $10^{-3}$ is applied. The objective here is to facilitate the acquisition process of appropriate upper and lower limits. After that, the smoothened curve is converted into absolute first difference (ABS) as shown Fig. 5. The closest peak on the left and right are automatically obtained and determined as lower limit, $I_{L}$ and upper limit, $I_{U}$.

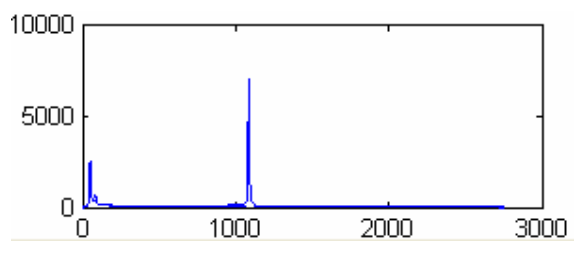

Fig. 4 Histogram of the Fig. 2 image

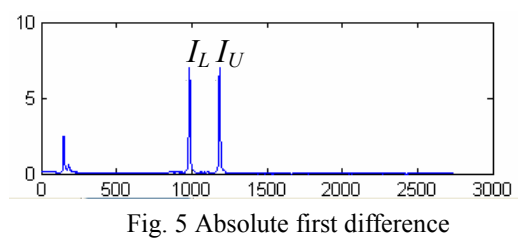

Lastly, the appropriate $I_{L}$ and $I_{U}$ are used for the linear contrast stretching as shown equation (1).

$$
F(i, j)=I_{\max } \frac{\left(I(i, j)-I_{L}\right)}{\left(I_{U}-I_{L}\right)} \ldots \ldots \ldots
$$

where $I_{\max }, I(i, j)$ and $F(i, j)$ denote the maximum intensity in the image, original image and contrast enhanced image respectively. After the contrast stretching, the visibility improved image is shown in the Fig. 6.

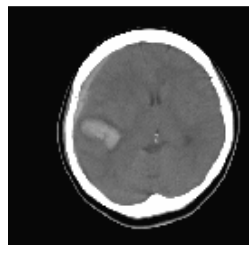

Fig. 6 Contrast improved image

\section{INTRACRANIAL EXTRACTION}

After contrast enhancement, intracranial area is extracted by alienating the background, skull and scalp from it. The alienation is done by using the thresholding method. The resulted binary image is shown in Fg. 7 . 


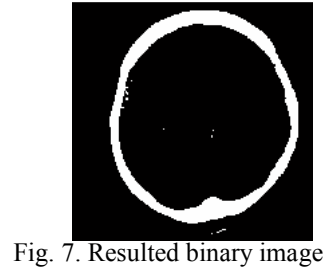

From the binary image, skull always appears to be the largest connected component. Therefore, in order to generate the intracranial mask, we detect the largest connected component and and fill up the hole inside the skull. Lastly, intensity of the skull is set to zero and the obtained intracranial area is shown in Fig. 8.

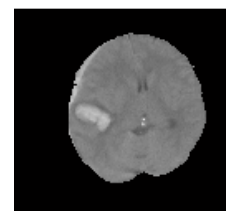

Fig. 8. Obtained intracranial area

\section{ROI CONTRAST ENHANCEMENT}

In order to ease the annotation process of hemorrhage, we adopt the priori knowledge to enhance the contrast of enhancement. In general, intensity of the intracranial hemorrhage is exceeded the peak position for the intensity. Therefore, the enhancement is focus on the higher intensity side to improve the visibility of the region of interest. Prior to the contrast enhancement, appropriate lower and upper limits should be determined. The steps are as below:

a) Construct the histogram for intracranial image.

b) Auto-identify the peak position to be the lower limit and upper limit is derived as below

$$
I_{U}=I_{L}+I_{\alpha}
$$

where $I_{\alpha}$ is predefined at 400 found from experimental observation.

c) Input the obtained $I_{L}$ and $I_{U}$ into equation (1) for the contrast stretching.

In order to reduce the "salt and pepper" noise appears in the enhanced image, median filter is employed. The image after the ROI visibility enhancement and "salt and pepper" noise reduction is shown in Fig. 9.

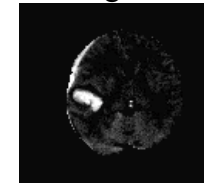

Fig. 9 Enhanced image

\section{POTENTIAL ROI CLUSTERING}

In this section, ROI contrast enhancement image is binarized to locate the hemorrhage. Due to the simplicity and speedy, thus, we considered the Otsu thresholding and k-means clustering.

For the k-means clustering, enhanced image is clustered into two clusters. We ignored the cluster with no ROI and considered the cluster with potential ROIs only. Besides hemorrhage, the cluster with potential ROIs also consists of some normal regions with intensity similar with hemorrhage. As a result, later on all potential regions will undergo decision-making process to acquire the right ROI.

The comparison results between Otsu thresholding and kmeans clustering are shown in Figure 10. From the obtained results, Otsu thresholding tends to produce noisy result compared with k-means method. Furthermore, the result from Otsu thresholding shows merging of hemorrhage with normal regions. This will cause the separation of ROI from normal regions to become impossible. As a result, k-means method is adopted.

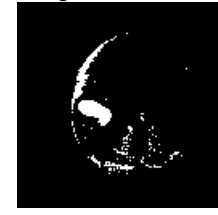

(a)

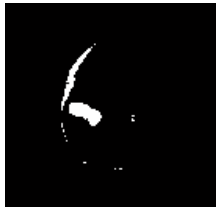

(b)
Fig. 10 Results obtained by (a) Otsu thresholding and (b) k-means clustering.

\section{FEATURE EXTRACTION AND DECISION MAKING PROCESSS}

Prior to feature extraction, all images are transformed into binary image. The function of the binarization here is to acquire all the connected components for normal and abnormal regions. Then the features are extracted from every connected component. The features use for detection of extra-axial hemorrhage and intra-axial hemorrhage are listed as in Table 1 and Table 2. The extracted features are used to differentiate the ROIs from non-ROIs. For extra-axial hemorrhagic detection, we take advantage of its location that is adjacent to the skull where more features can be adopted. Based on the acquired database, for extra-axial hemorrhage we considered epidural hematoma and subdural hematoma and for intra-axial hemorrhage we considered all cerebral hemorrhages. Other bright regions such as sinus, vessel, midline falx and white brain matter are classified as normal region.

A decision-making model is created as shown in Fig. 11 in order to annotate the extra-axial hemorrhage, intra-axial hemorrhage and non-ROI. Firstly, the potential ROI is distinguished by determining whether the adjacent neighbour is skull or not. If the adjacent neighbour is skull then it proceeds with the extra-axial hemorrhagic decision making process. Otherwise, it continues with the intra-axial hemorrhagic decision making process.

TABLE 1

FEATURES FOR INTRA-AXIAL HEMORRHAGE DETECTION

\begin{tabular}{|l|l|}
\hline Feature & Remarks \\
\hline Region area & $\begin{array}{l}\text { The number of pixels in the region of } \\
\text { the enhanced image in Section VI. }\end{array}$ \\
\hline Region mean & $\begin{array}{l}\text { The average of pixels in the region of } \\
\text { the original image in Section VI. }\end{array}$ \\
\hline Solidity & $\begin{array}{l}\text { The proportion of the pixels in the } \\
\text { convex hull. }\end{array}$ \\
\hline
\end{tabular}


TABLE 2

FEATURES FOR EXTRA-AXIAL HEMORRHAGE DETECTION

\begin{tabular}{|l|l|}
\hline Feature & Remarks \\
\hline Contact area & $\begin{array}{l}\text { The number of pixels in the region contact with the } \\
\text { border. }\end{array}$ \\
\hline Region area & $\begin{array}{l}\text { The number of pixels in the region of the enhanced } \\
\text { image in Section VI. }\end{array}$ \\
\hline Region mean & $\begin{array}{l}\text { The average of pixels in the region of the original } \\
\text { image in Section VI. }\end{array}$ \\
\hline Solidity & The proportion of the pixels in the convex hull. \\
\hline Orientation & The angle in degree. \\
\hline Eccentricity & $\begin{array}{l}\text { The value is between } 0 \text { and } 1 . \text { An ellipse whose } \\
\text { eccentricity is } 0 \text { is actually a circle, while an ellipse } \\
\text { whose eccentricity is } 1 \text { is a line segment. }\end{array}$ \\
\hline Major axis length & Longest diameter \\
\hline
\end{tabular}

The parameters such as I $\boldsymbol{a}, \mathrm{I} \boldsymbol{m}$ and Is represent the threshold value for the area, mean and solidity for intra-axial hemorrhage respectively. Whereas Eca, Ea, Em, Ecc, Eo, Es and Emaj represent the threshold value for contact area, region area, mean, eccentricity, orientation, solidity and major axis length for extra-axial hemorrhage respectively. The major reason of employment of this decision-making is due to its flexibility in updating the suitable threshold values and simplicity in the implementation.

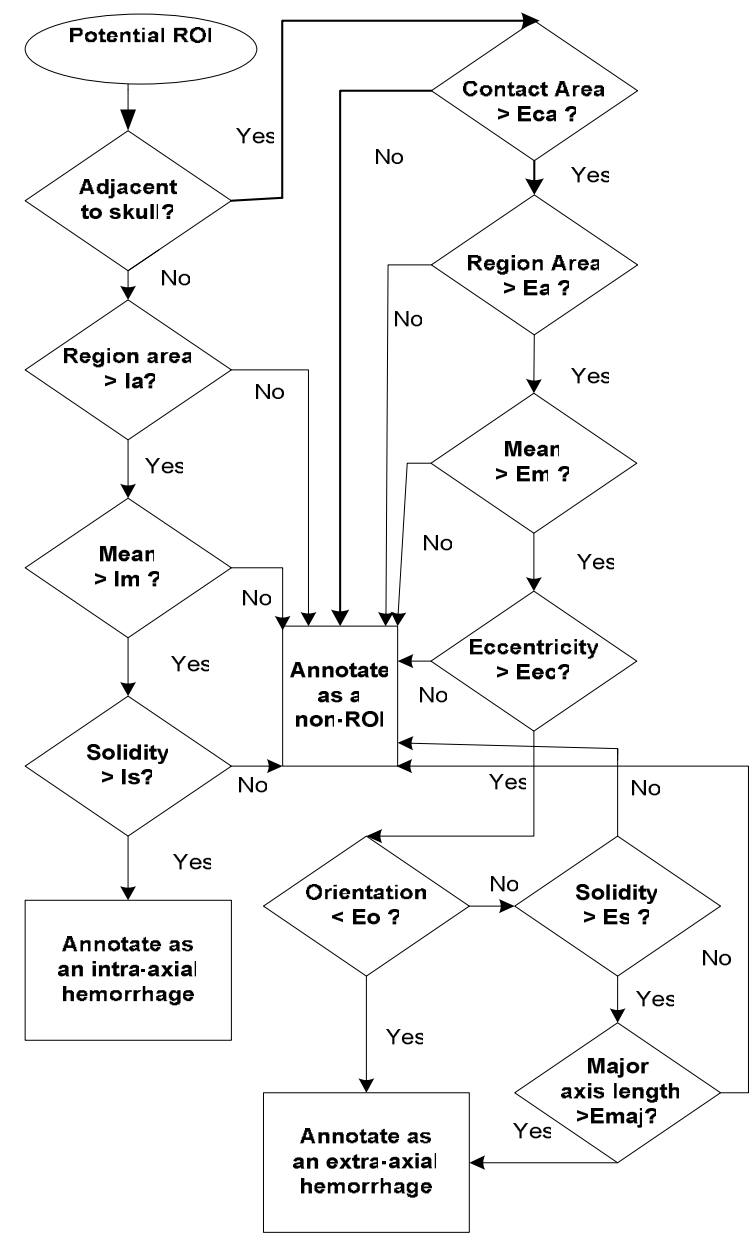

Fig. 11 Decision-making model

\section{EM SEGMENTATION}

EM segmentation at this stage is to segment the image after excluding the hemorrhage into CSF and brain matter. The EM algorithm is an iterative method to compute the maximum likelihood estimate in the presence of missing or hidden data.

Every iterations of EM segmentation consists of expectation (E) step and maximization (M) step. In E step, the probability density function for a Gaussian distribution as in equation (2) is used to compute the cluster probability for every pixel.

$f_{i}\left(x \mid \theta_{i}\right)=\frac{1}{(2 \pi)^{d / 2}\left|\sum_{i}\right|^{1 / 2}} \exp \left[-\frac{1}{2}\left(x-\mu_{i}\right)^{t} \sum_{i}^{-1}\left(x-\mu_{i}\right)\right]$

where $\theta_{i}=\left(\mu_{i}, \Sigma_{i}\right), \mathrm{x}$ is a d-dimensional feature vector, $\mu_{i}$ is the mean vector and $\sum_{i},\left|\sum_{i}\right|$ and $\sum_{i}^{-1}$ are the $\mathrm{d} \times \mathrm{d}$ covariance matrix, its determinant and inverse respectively.

In $M$ step, the probability values obtained in E-step is used to re-compute the means, covariances and mixing weights. The results of segmentation for Fig. 6 are shown in Fig. 12. The results of segmentation can be further used for detection of abnormalities in ventricles, for example.

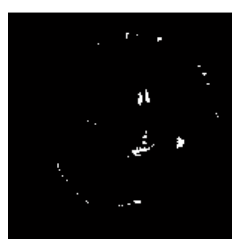

(a)

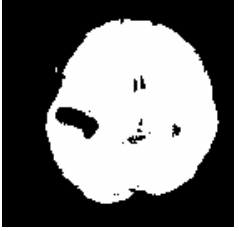

(b)
Fig. 12 Segmentation results for (a) CSF and (b) brain matter.

\section{EXPERIMENTAL RESULTS AND DISCUSSION}

The CT head images with dimension 512 X 512 are provided by the collaborating hospital. The images on 31 patients have been tested and for each patient few images are being taken. Overall, in total there are 244 potential hemorrhagic regions. In this section, all the detection results will be discussed. Some of the experimental results of detection for extra-axial hemorrhage and intra-axial hemorrhage are shown in Fig. 13.

Actually, in medical aspect, recall rate is more significant than precision. This is because recall rate only focuses on the correctly classified hemorrhagic regions. The correctly classified hemorrhagic regions are useful to the doctors rather than misclassified hemorrhagic regions. However, the detection results are evaluated in terms of recall and precision. The results are shown in Table 3. For the classification, recall and precision are defined as:

$$
\text { Recall }=
$$

Total correctly classified hemorrhagic regions

$$
\text { Total actual hemorrhagic regions }
$$


Precision $=$

Total correctly classified hemorrhagic regions

Total correctly classified hemorrhagic regions + Total misclassified hemorrhagic regions
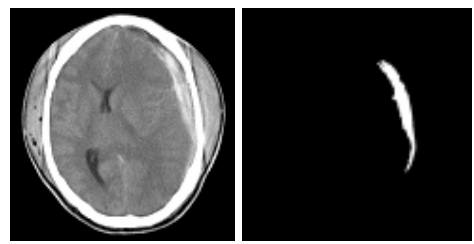

(a) Extra-axial hemorrhage's image (b) Obtained result for (a)
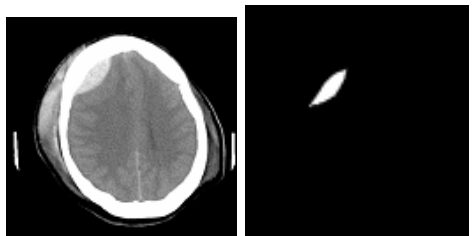

(c) Extra-axial hemorrhage's image (d) Obtained result for (c)
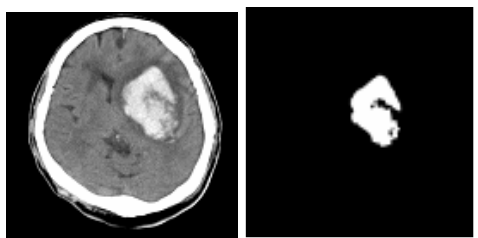

(e) Intra-axial hemorrhage's image (f) Obtained result for (e)
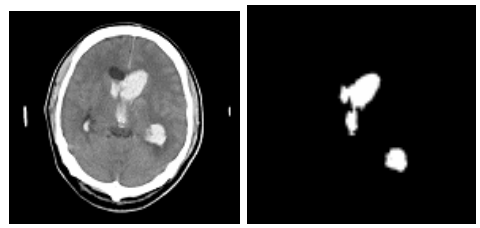

(g) Intra-axial hemorrhage's image (h) Obtained result for (g)

Fig. 13 Experimental results

TABLE 3

DETECTION RESULTS

\begin{tabular}{|l|l|l|}
\hline $\begin{array}{l}\text { Type of } \\
\text { hemorrhage }\end{array}$ & Recall & Precision \\
\hline Intra-axial & 0.8043 & 0.8880 \\
\hline Extra-axial & 0.9245 & 0.9333 \\
\hline
\end{tabular}

From Table 3, the recall rate for extra-axial hemorrhage is higher as more features can be adopted to detect the extraaxial hemorrhage in order to obtain better results. However, there are still some difficulties in identifying extra-axial hemorrhage as bright regions like sinus also have similar intensity as extra-axial hemorrhage. The recall rate for intraaxial hemorrhage is lower as some of the intra-axial hemorrhages are located very near to the boundary of the intracranial area. Due to the imperfectness of the segmentation, these hemorrhagic regions have merged with the boundary and are only being used in extra-axial hemorrhagic decision making process.

The precision rate for extra-axial hemorrhage is higher as the contact area feature adopted at the first level has filtered out non-ROIs such as falx to avoid them being misclassified as hemorrhagic regions. We adopted the priori knowledge where the contact area with the boundary for subdural and epidural hemorrhages is more than falx. In addition to this, other features that were used for the following decision making also have reduced the misclassification cases. The precision for intra-axial hemorrhage is lower is due to the occurrence of more misclassification cases. Some bright regions such as falx and calcification are very hard to be differentiated from the intra-axial hemorrhage.

\section{COnClusions}

We have presented our approach on the detection of extraaxial and intra-axial hemorrhages. The method yielded $93.33 \%$ precision and $92.45 \%$ recall for intra-axial hemorrhagic detection. Whereas extra-axial hemorrhagic detection, it achieved $80.43 \%$ recall and $88.80 \%$ precision. Plans for future work include adoption of more features especially for intra-axial hemorrhagic detection and the implementation of more annotations such as infract and some abnormalities in ventricles.

\section{REFERENCES}

[1] Dubravko Cosic, Sven Loncaric, Rule-Based Labeling of CT Head Image, 6th Conference on Artificial Intelligence in Medicine, 1997, pp. 453-456

[2] Matesin Milan, LONCARIC Sven, PETRAVIC Damir, A rule-based approach to stroke lesion analysis from CT brain images, 2nd international symposium on image and signal processing and analysis, June 2001, pp. 219-223.

[3] Y.Liu, N.A.Lazar, W.E.Rothfus, F.Dellaert, A.Moore, J.Schneider, and T.Kanade, Semantic-based Biomedical Image Indexing and Retrieval, Trends and Advances in Content-Based Image and Video Retrieval, Shapiro, Kriege, and Veltkamp, ed. February, 2004.

[4] T.Hara, N.Matoba, X.Zhou, S.Yokoi, H.Aizawa, H.Fujita, K.Sakashita and T.Matsuoka, Automated Detection of Extradural and Subdural Hematoma for Contentenhanced CT Images in Emergency Medical Care, Proceeding of SPIE, 2007.

[5] T.Chan, Computer aided detection of small acute intracranial hemorrhage on computer tomography of brain, Computerized Medical Imaging and Graphics 07(4-5): 285-298, June-July, 2007.

[6] Petra Perner, An Architecture for a CBR Image Segmentation System, In Proceedings of ICCBR, 1999, pp. 525-534.

[7] K.H. Hohne and W.A. Hanson, Interactive 3D segmentation of MRI and CT volumes using morphological operations, J. Comp. Assist. Tomogr. 2, 1992, pp. 285-294.

[8] Qingmao $\mathrm{Hu}$, Guoyu Qian, Aamer Aziz, Wieslaw L. Nowinsk, Segmentation of brain from computed tomography head images, Proceedings of IEEE Engineering in Medicine and Biology $27^{\text {th }}$ Annual conference, Sept. 2005, pp. 3375 - 3378.

[9] N. A. Mohamed, M. N. Ahmed, and A. Farag, Modified Fuzzy CMean in Medical Image Segmentation, Acoustics, Speech, and Signal Proceedings, vol.6,1999, pp. 3429-3432.

[10] Wen-Nung Lie, Wen-Hung Peng, Cheng-Hung Chuang, Efficient content-based CT brain image retrieval by using region shape features, ISCAS (4), 2002, pp. 157-160.

[11] Tong Hau Lee, Mohammad Faizal Ahmad Fauzi and Ryoichi Komiya, Segmentation of CT Brain Images Using K-means and EM Clustering, Fifth International Conference on Computer Graphics, Imaging and Visualization, 2008, pp. 339-344. 